\title{
Design of a WSN System for Condition Monitoring of the Mechanical Equipment with Energy Harvesting
}

\author{
http://dx.doi.org/10.3991/ijoe.v11i2.4366 \\ Kai Zheng, Yun Zhang, Bo Chen, Yanfang Dong, Tianliang Li, Yang Wang \\ Wuhan University of Technology, Wuhan, China
}

\begin{abstract}
Design of a wireless sensor network (WSN) system for mechanical condition monitoring (WSN-MCM) is presented in this paper. The main purpose of this study is to develop a WSN system for mechanical condition monitoring as the existing mature WSN nodes are not suitable for long-time monitoring of mechanical equipment due to their short working life and low acquisition accuracy. To realize long-time working of the sensor nodes, we designed an energy harvesting board to harvest the energy and transfer energy with high efficiency. Meanwhile, the nodes have the function of high-accuracy acquisition of analog signals and can access various low-frequency and high-frequency sensors. The experiment results demonstrate that the WSN system designed is suitable for mechanical condition monitoring.
\end{abstract}

Keywords-WSN-MCM, Energy harvesting, High accuracy data acquisition, Long term working

\section{INTRODUCTION}

Safety operation of mechanical equipment is of great significance to modern industrial production. The mechanical condition monitoring (MCM) method was proposed to maintain the health of mechanical equipment. Mechanical equipment is usually monitored through wired monitoring system. Wired monitoring technology has come of age till now. It has been widely used in complicated and key mechanical equipment such as large rotating machinery, harbor machinery and aeronautical facilities, etc.

There are many problems as the wired method is adopted to monitor large mechanical equipment, including complex wiring, high cost installation and difficulty in sensor layout $[1,2]$. And the maintenance cost of a wired monitoring system is high as the wired cable is easy to age. Furthermore, it is difficult to collect data through wired means on some special occasions, for example, when acquiring the physical parameter of a rotating shaft. WSN-MCM is a solution to these problems. At present, many commercial sensor network nodes such as MICA series and Telos nodes cannot realize high-precision data acquisition and do not have long-term working capability. It is unsuitable to use them for long-term mechanical condition monitoring. So, it is indeed important to design WSN nodes.

WSN technology promise huge potentials for application in mechanical condition monitoring $[3,6]$. In the field of mechanical condition monitoring based on WSN, many researchers focus their attention on problems such as sensor node processing [1], fault diagnosis of mechanical equipment using WSN protocols[1,2,4] or reducing the energy consumption of sensor nodes with intelligent methods $[1,2]$. In this paper, we focus on the hardware and software design of the WSN system and the long-term working ability of sensor nodes.

The remainder of this paper is organized as follows. We put forward a WSN system for mechanical condition monitoring and demonstrated the hardware and software design of the WSN system in Section 2. In Section 3, we did experiments to evaluate the performance of the WSN system. Conclusions were drawn in Section 4.

\section{SYSTEM ARCHITECTURE AND NETWORK COMPONENTS}

\section{A. System Architecture}

This paper has presented a WSN system for mechanical condition monitoring, as shown in Figure 1. This is a hierarchical system, including sensors, WSN nodes, sink nodes, central supervisory station and local area networking. Specifically, sensors mainly acquired physical quantities in mechanical condition monitoring; sensor nodes processed signals, acquired data with high precision, and sent data to the base station; the base station fused data and sent the data to the central supervisory station; and the central supervisory station stored and analyzed data, monitored the condition and diagnosed the fault of the machine, exchanged and shared data with other computers within a local area network.

\section{B. Design of the Wireless Sensor Nodes}

In the WSN system designed for mechanical condition monitoring, the sensor nodes should be able to: (1) acquire and process the data from the sensors and compensate (including hardware and algorithm methods) the precision of the sensors; (2) pre-process the data acquired and transmit the data pre-processed to the coordinator; and (3) work for a long time.

To achieve modular design and reduce the size of the sensor node board, the nodes we designed consist of three vertically-stacked circuit boards. Specifically, the first circuit board responsible for energy harvesting stores and manages the energy harvested and supplies power for other modules of the sensor nodes. Meanwhile, as the circuit board for energy harvesting has a backup source, it can automatically switch to the backup battery when it fails to harvest enough energy so that the sensor network nodes can keep working; the second circuit board is re- 
PAPER

Design Of A WSN System For Condition Monitoring Of The Mechanical EquiPment With...

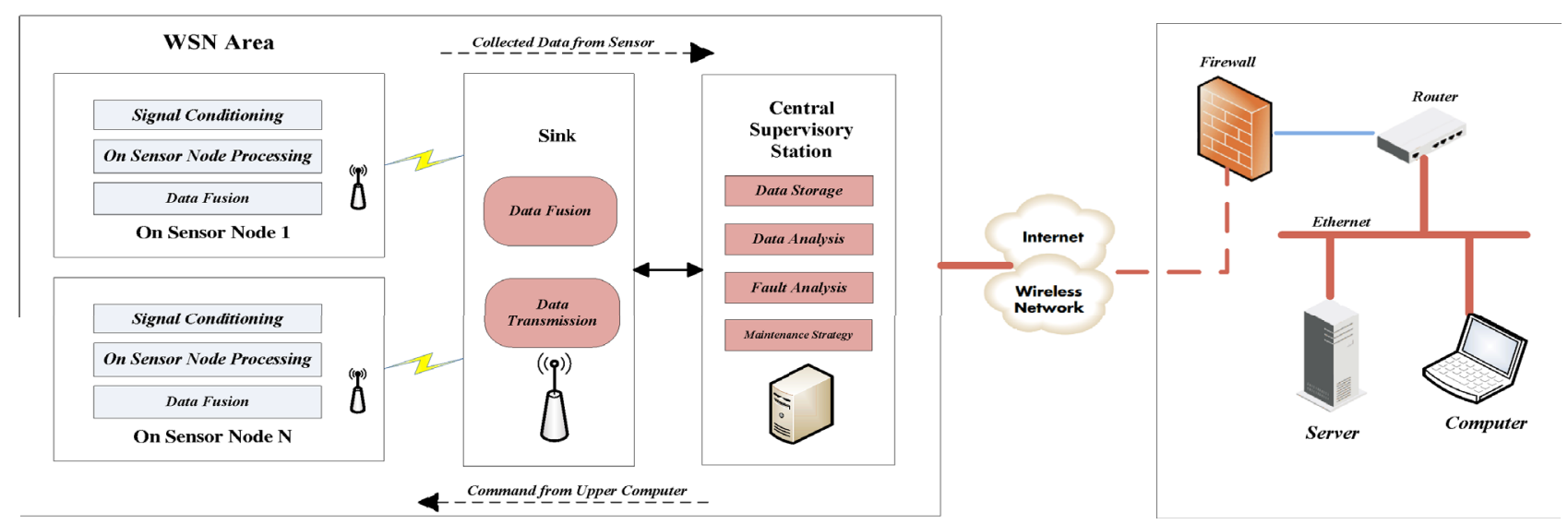

Figure 1. Architecture of WSN for mechanical condition monitoring

sponsible for conditioning and acquiring signals from theanalog and digital sensors; and the third circuit board is responsible for sensor node data processing so as to realize data fusion, algorithmic calculation and wireless communication. The three circuit boards are connected by the connector.

\section{Power Supply Board}

Power supply is a critical link of the sensor network nodes. Long-time work of the wireless nodes is very important in WSN-MCM. In traditional WSN system, nodes are usually powered by batteries and cannot work for a long time [7, 8, 9]. Therefore, we designed the power supply board of the nodes, which can support energy harvesting, as shown in Figure 2. It can:

1. Harvest the energy from the sensor network nodes, such as solar energy and vibration energy.

2. Realize maximum energy harvesting with Maximum Power Point Tracking (MPPT) algorithm.

3. Store the energy harvested and have backup source. When the conditions for energy harvesting are changed, achieving automatic switch between energy storage unit (e.g., super-capacitor) and backup sources.

The power supply board of the nodes we designed comprises three parts, namely, energy harvesting, power management and storage and power regulation. This node board collects solar energy or vibration energy, harvests energy via the energy harvesting and management chip BQ25505, and stores the energy in the super-capacitor $(5.5 \mathrm{~V} / 1 \mathrm{~F})$. In case that the energy harvesting conditions change, the power supply board is also equipped with a backup battery with a capacity of $3000 \mathrm{mAH}$ so as to ensure long-time power supply for the nodes. When the voltage of the rechargeable battery was greater than the threshold voltage in the energy harvesting and management chip BQ25505, low-level signal was produced in the pin VB_SEC_ON of BQ25505 and high-level signal was produced in VB_PRI_ON. Then, the two signals were loaded to PMOS, so that the rechargeable battery could drive the load. On the contrary, when the voltage of the rechargeable battery was lower than the threshold voltage, the backup battery was used to drive the load. Then, the power supply unit could achieve automatic switch, with the switching time lower than $10 \mathrm{~ms}$. The switch control circuit is shown in Figure 3.

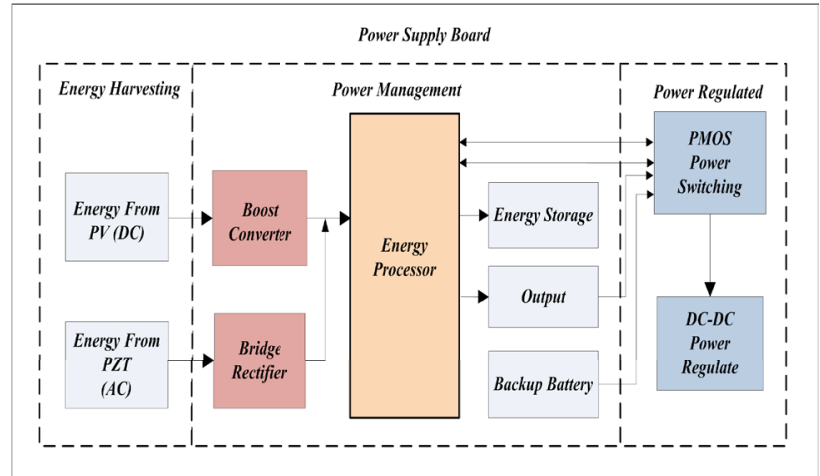

Figure 2. System diagram of power supply board

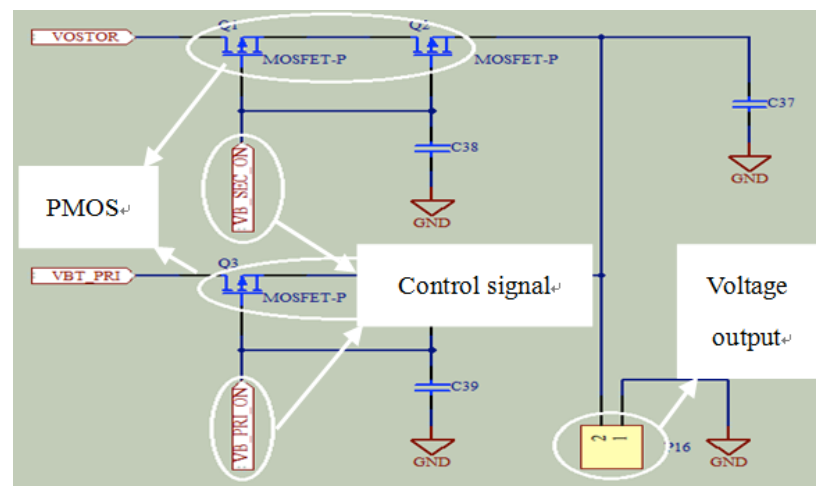

Figure 3. Power switching control circuit of the power supply board

\section{The Processing and Communication Board}

The processing and communication main-board mainly comprises the following units: main control chip and external module extension unit, communication unit. The sensor nodes we designed could be used for highprecision acquisition of analog and digital signals.

In design of the signal processing and communication main-board, we used MSP430F149 as the main control chip to acquire data and realize precision compensation of the sensors. MSP430F149 is a 16-bit micro-controller. It has the following merits [5]: (1) MSP430F149 has several low power modes. In power down mode, it only consumes $0.1 \mathrm{uA}$ electric current. In $8 \mathrm{MHZ}$ normal operation, the current it consumes is only 200-400uA. (2) MSP430F149 has abundant peripheral units (including serial ports and I2C), and 64 IO ports, which are helpful for extension of peripheral units. (3) MSP430F149 has a hardware multi- 
PAPER

Design Of A WSN System For Condition Monitoring Of The Mechanical EquiPment With...

plier which can meet specific requirements (e.g., FFT algorithm) for sensor node data processing.

We consider using an external AD chip to realize precise measurement of physical parameters like vibration and displacement. Given the power consumption of $\mathrm{AD}$ chip and the resolution needed for the external sensors, we adopted a 24-bit external AD conversion chip ADS1256 and used $2.5 \mathrm{~V}$ external precision reference voltage source ADR421. As an acquisition chip which can sample analog signals simultaneously, ADS1256 is powered by $5 \mathrm{~V}$ analog power supply and exchanges data with MSP430F149 through SPI agreement.

Several commercial communication units and chips were compared in order to select an appropriate wireless communication unit. All the wireless communication units support Standard IEEE 802.15.4, we chose XBee modules as they have ZigBee protocol stack and feature low-cost and low power consumption. Furthermore, data transmission between ZigBee and MSP430 is achieved via UART protocol (Tx current: $45 \mathrm{~mA}$ ) and long-range communication.

\section{E. The Sensor Board}

The sensor board mainly comprises signal conditioning circuit and sensors attached to the circuit board, and provide expansion interfaces to support access of various low-frequency and high-frequency external sensors (e.g., eddy current sensor and capacitance sensor used in mechanical monitoring and measurement). The sensors attached to the sensor board include MEMS acceleration sensor, tilt angle sensor and temperature sensor.

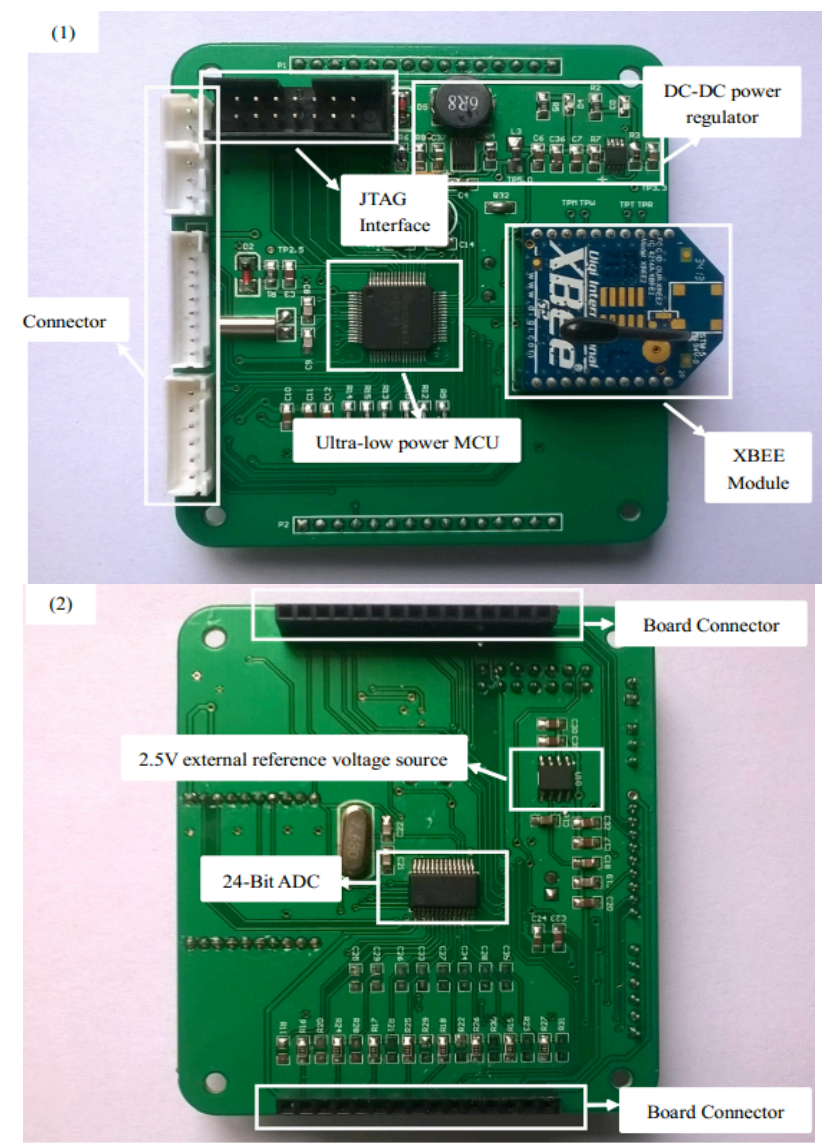

To acquire the signals from these sensors, we set four channels for collecting analog signals and four channels for collecting digital signals. Before the sensors were connected to the channels, the signals from them should be conditioned. Following is a detailed description about the sensor signal conditioning circuit on the sensor board.

Eddy current sensor is a kind of typical precision sensor, which is widely used in the field of machinery. In mechanical condition monitoring, eddy current sensor is usually used to acquire vibration or displacement signal. Its output voltage or current signals have a linear relationship with displacement, which can be acquired by $\mathrm{AD}$ port.

MEMS accelerometer is ADXL345, which is a three axis accelerometer used to acquire acceleration vibration signals in this system. With an SPI digital output port, it exchanges data with the main board via SPI bus. The physical map of WSN nodes is shown in Figure 4.

\section{F. Base Station}

The base station plays an important role in the WSNMCM system since it coordinates the communication activity among the wireless nodes and serves as a gateway between the local system and the upper computer. The base station comprised power supply module, computer interface module, main control module and wireless communication module. It received the data collected by WSN nodes and sent them to the upper computer. All the data were saved by SQL database in the upper computer. The database stored the relevant properties of the system nodes (including Node ID, Sensor data, time and Node Signal Strength).

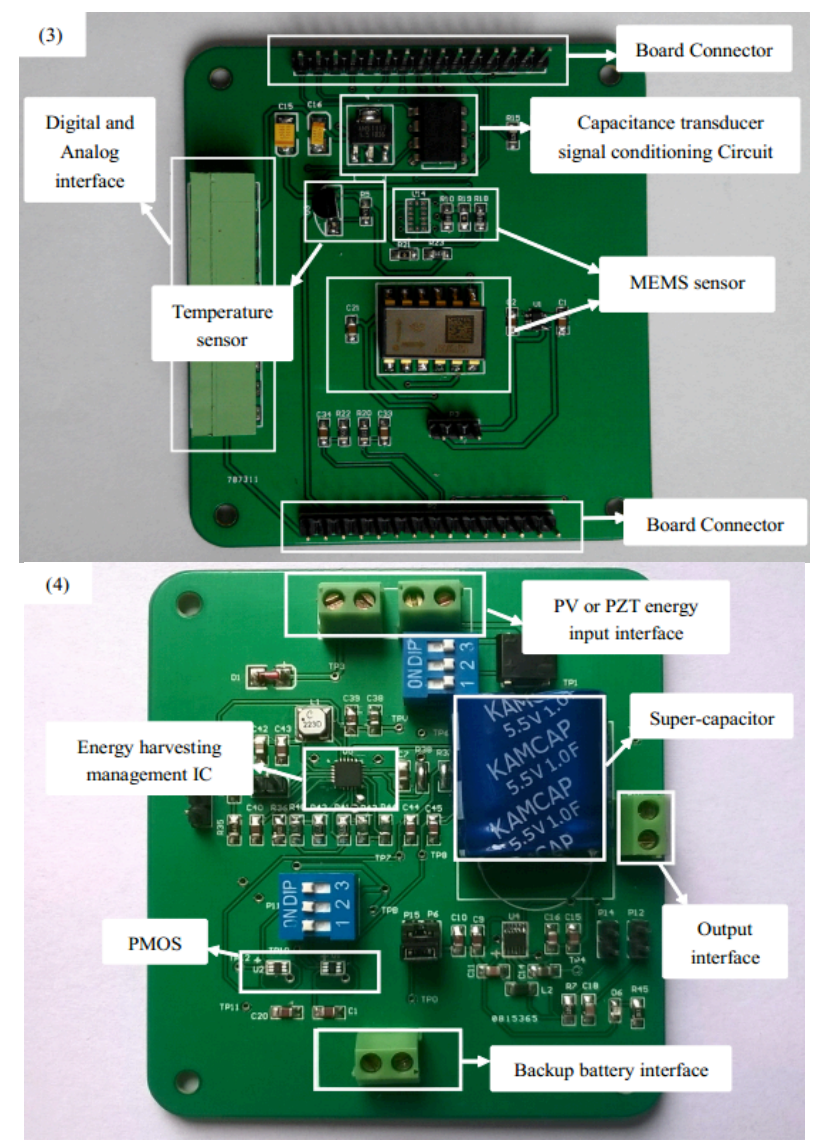

Figure 4. Physical map of WSN nodes: (1) Processing and communication board [upper side] (2) Processing and communication board [lower side] (3) Sensor board (4) Power-supply board 


\section{G. Building of System Network}

The wireless protocol between nodes was based on IEEE802.15.4/ZigBee. In this Protocol, nodes were divided into coordinator, router and terminal node. The function of the coordinator (also called base station) was to build a sensor network and configure the network, and the routers allowed the nodes to join the network and facilitated the communication among child nodes while the terminal nodes joined the network. The process of data transmission is as follows: When the sensor network switched into data transfer mode, the coordinator scanned the physical channel to determine whether there was network on this channel and recorded its signal strength. After channel scan, it selected a channel with the smallest signal strength to build a network. After the network was built, the coordinator used $0 \mathrm{X} 0000$ as its network address. When the routers and the terminal nodes asked to join the network, the coordinator would provide them with a unique 16-bit network address according to node type and address distribution algorithm. When the router booted up, it first scanned the existing network, and then selected a coordinator and asked to join the network. The terminal nodes could only join the network of routing nodes or terminal nodes.

Upper computer software was designed for WSNMCM to acquire data from nodes, and store the data into database. It comprised three modules: (1) System configuration module, which mainly configured the system parameters and communicated with the base station. (2) Data acquisition process control module, which mainly controlled the process of data acquisition, realized automatic storage of data and displayed the topology between WSN nodes. (3) Data processing module, which realized online and offline data processing. Figure 5 shows the interface of the software.

\section{EXPERIMENT RESULTS}

\section{A. WSN Node Sampling Accuracy Verification}

We did a vibration measurement experiment in order to test the sampling accuracy of the sensor nodes of the flywheel rotor on the test platform for large rotary machine, with the physical map shown in Figure 6. Firstly, the eddy current sensor (the parameters were shown in Table 2) was fixed on the adjustable support and the signals from the probe of the eddy current sensor were sent to the transmitter; Secondly, the signals were sent to the $\mathrm{PC}$ via the LabView data acquisition system and NI-USB6351 acquisition card. Then, their respective zero point positions were determined according to fitted linear equation of the eddy current sensor (current between the two reference points: $12 \mathrm{~mA}$ ). Their reference zero point positions were obtained after the distance between the sensors and the wheel disc was changed by adjusting the micrometer behind the fixed mount of the sensor. We set the experi-

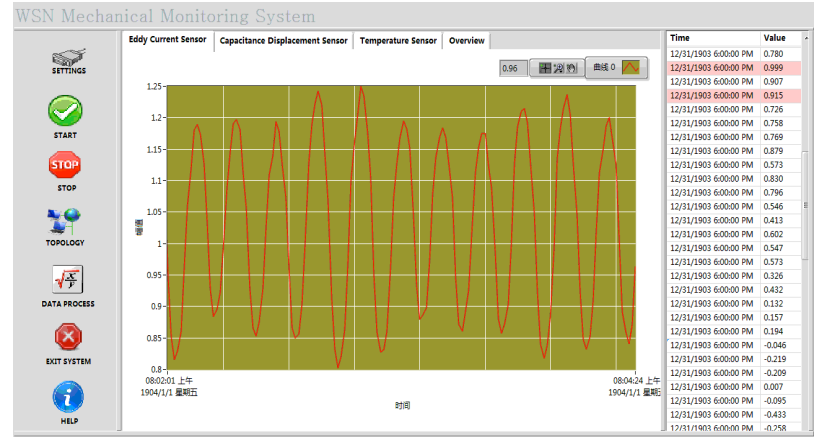

Figure 5. Display interface of the software for WSN-MCM

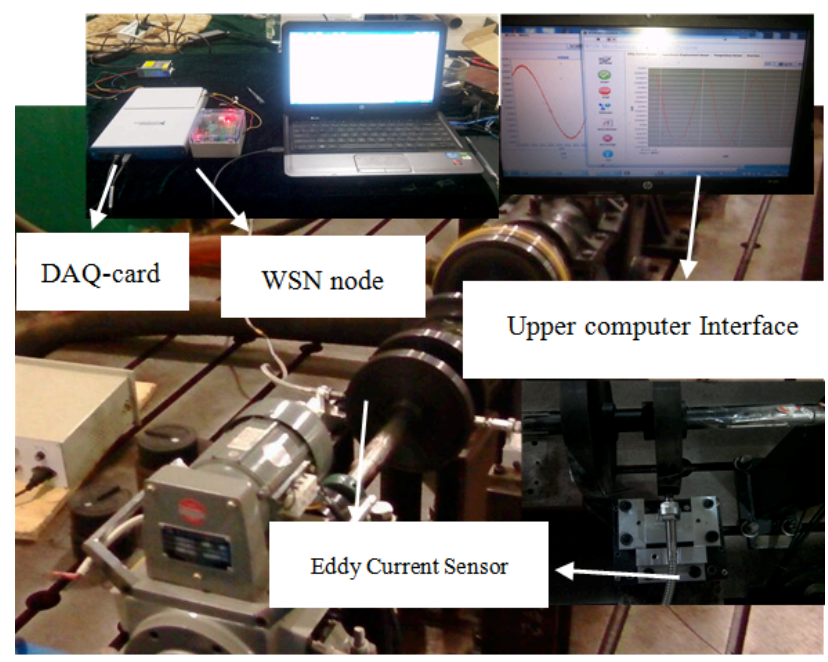

Figure 6. Experiment for accuracy comparison between the WSN nodes and the acquisition card

mental linear zero of the eddy current displacement sensor at $1.58 \mathrm{~mm}$. Finally, the motor control box was adjusted to reach the preset rotating speed so that the rotating shaft could work at different constant speeds in the experiment.

We made a spectrum analysis on the data acquired by the WSN nodes and the acquisition card from the eddy current sensors respectively when the motor rotated at a speed of around 300r/min, 600 $/ \mathrm{min}$ and $900 \mathrm{r} / \mathrm{min}$. The results were shown in Figure 7. The main frequencies of the two after FFT are shown in Table 1. From the table, we can know that the acquisition frequencies of the two were basically the same and the accuracy of WSN nodes was higher. Moreover, as it is known that the rotors were misaligned, double frequency should appear after the spectrum analysis was made on the data acquired. After FFT of the data acquired by the acquisition card and WSN nodes, double frequency could be observed in the spectral diagram. From Table 1, it can be found that the sampling accuracy of WSN nodes is higher than the NI acquisition card, which shows that the nodes we designed conform to the requirements of mechanical condition monitoring.

TABLE I.

COMPARISON OF FREQUENCY AND ERROR BETWEEN WSN NODES (WIRELESS) AND NI ACQUISITION CARD (WIRED)

\begin{tabular}{|c|c|c|c|c|c|c|c|c|c|c|}
\hline & \multicolumn{3}{|c|}{ 300r/min(5HZ) } & \multicolumn{3}{c|}{ 600r/min(10HZ) } & \multicolumn{3}{c|}{ 900r/min(15HZ) } \\
\hline & Derivation & Wireless & Wired & Derivation & Wireless & Wired & Derivation & Wireless & Wired \\
\hline 1st freq. (Hz) & 5.000 & 5.000 & 4.944 & 10.000 & 10.001 & 9.983 & 15.000 & 14.993 & 14.600 \\
\hline 2 st freq. (Hz) & 10.000 & 10.000 & 9.888 & 20.000 & 20.006 & 20.102 & 30.000 & 29.032 & 28.602 \\
\hline Error of 1st freq. (\%) & - & 0 & 1.12 & - & 0.01 & 0.17 & - & 0.05 & 2.26 \\
\hline
\end{tabular}


PAPER

DESIGN Of A WSN System For CONDITION MONITORING Of THE MECHANICAL EQUiPMENT With...
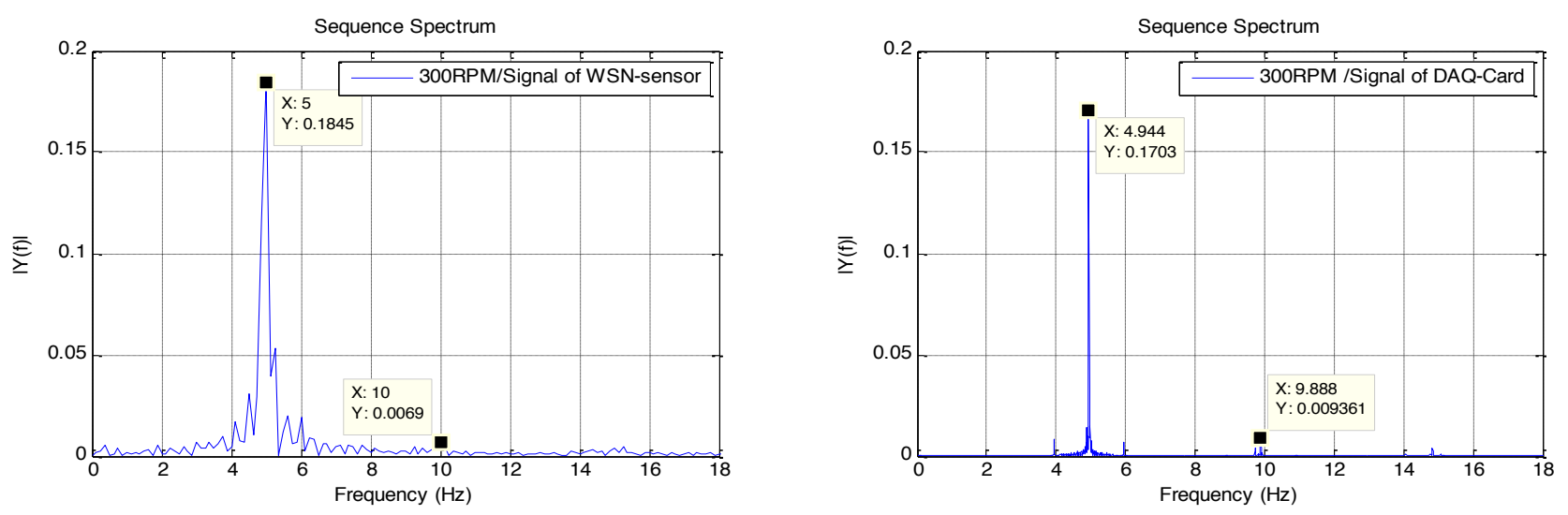

Figure 7. Spectrograms of eddy current acquired by WSN nodes and acquisition card at a speed of $300 \mathrm{r} / \mathrm{min}$

\section{B Energy Harvesting Performance Evaluation}

We used a 93.5-cm2 PV module which provided a maximum power of $420 \mathrm{~mW}$ and a backup battery with a capacity of $3000 \mathrm{mAH}$ to supply energy for the WSN nodes. To test the power switch function of the power supply board, we designed the following experiment which is shown in Figure 8, and the experiment result is shown in Figure 9. As shown in Figure 9, during the experiment, when the PV module was lit under about 500 LUX condition, the control signal of PMOS VB SEC ON changed to low level, while the signal of VB PRI ON changed to high level, so that the rechargeable battery could drive the load. When the PV module was not lit, the backup battery was used to drive the load.

\section{CONCLUSION}

In this paper, we designed and developed a WSN system for mechanical condition monitoring with energy harvesting. The designed nodes have the function of energy harvesting and support switching between power supply units. The methods of high-efficiency energy transferring and energy harvesting were employed, so as to realize long-time monitoring with comprehensive means. In addition, the nodes have high-precision AD sampling resolution and can support the access of various low-frequency and high-frequency sensors and MEMS sensors. These sensors are usually applied in mechanical condition monitoring.

The results of the experiment on the WSN system show that the designed nodes support long-time monitoring and high sampling precision. Meanwhile, due to their low cost, they are suitable for large-scale deployment and can be used for condition monitoring of mechanical equipment.

\section{REFERENCES}

[1] Hou, Liqun, and Neil W. Bergmann. Novel industrial wireless sensor networks for machine condition monitoring and fault diagnosis. Instrumentation and Measurement, IEEE Transactions on 61.10 (2012): 2787-2798.

[2] Hou, L., and N. W. Bergmann. A novel industrial wireless sensor network for condition monitoring and fault diagnosis of electrical machines. Australian Journal of Electrical and Electronics Engineering 10.4 (2013): 505-514. http://dx.doi.org/10.7158/E12170.2013.10.4

[3] Ballal, P., et al. Mechanical fault diagnosis using wireless sensor networks and a two-stage neural network classifier. Aerospace conference, 2009 IEEE. IEEE, 2009.

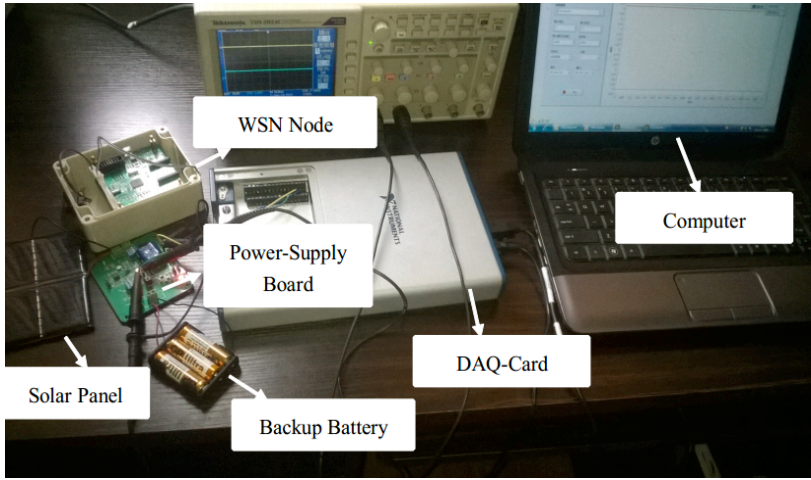

Figure 8. Experiment of WSN nodes' function of power switching
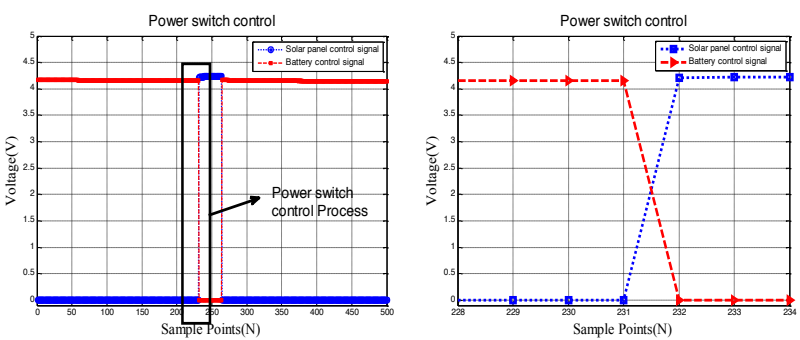

Figure 9. Test result of the power switch control ( when the PV module was lit under about 500 LUX condition, the control signal of solar panel changed to low level, while the control signal battery changed to high level)

[4] Lu, Bin, and Vehbi C. Gungor. Online and remote motor energy monitoring and fault diagnostics using wireless sensor networks. Industrial Electronics, IEEE Transactions on 56.11 (2009): 46514659.

[5] Wei Yang, Ping Wang. Feature and application of the MSP430 serial ultra-low-power MCU. Foreign Electronic Measurement Technology 12 (2008): 017.

[6] Hashemian, H. M. Wireless sensors for predictive maintenance of rotating equipment in research reactors. Annals of Nuclear Energy 38.2 (2011): 665-680 http://dx.doi.org/10.1016/j.anucene.201 0.09 .012

[7] Flammini, Alessandra, et al. Wired and wireless sensor networks for industrial applications. Microelectronics Journal 40.9 (2009): 1322-1336. http://dx.doi.org/10.1016/j.mejo.2008.08.012

[8] Magno, Michele, et al. Extended Wireless Structural Monitoring Through Intelligent Hybrid Energy Supply. IEEE Transactions on Industrial Electronics, (2014): 1-1.

[9] Zielinski, M., et al. Design of a low power wireless sensor network node for distributed active vibration control system. Microelectronics and Electronics (PRIME), 2014 10th Conference on Ph. D. Research in. IEEE, 2014. 
[10] Haase, Jan, Javier Moreno Molina, and Dietmar Dietrich. Poweraware system design of wireless sensor networks: Power estimation and power profiling strategies. Industrial Informatics, IEEE Transactions on 7.4 (2011): 601-613.

\section{AUTHORS}

Kai Zheng is with the School of Mechanical and Electronic Engineering, Wuhan University of Technology, Wuhan, China.

Yun Zhang is with the School of Mechanical and Electronic Engineering Wuhan University of Technology, Wuhan, China.

Bo Chen is with the School of Mechanical and Electronic Engineering, Wuhan University of Technology, Wuhan, China.
Yan-Fang Dong is with the School of Mechanical and Electronic Engineering, Wuhan University of Technology, Wuhan, China.

Yang Wang is with the School of Mechanical and Electrical Engineering, Wuhan University of Technology, Wuhan, China.

Tianliang Li is with the School of Mechanical and Electrical Engineering, Wuhan University of Technology, Wuhan, China.

This research is supported by Hubei Digital Manufacturing Key Laboratory, China. Submitted 06 January 2015. Published as resubmitted by the authors 10 March 2015 . 